\title{
INFLUÊNCIA DO TEMPO DE TABAGISMO NOS SINAIS VITAIS
}

Camila Aguiar Santiago, Dionei Ramos, Rebeca Nunes Silva, Juliana Tiyaki Ito, Juliana Souza Uzeloto, Fabiano Francisco de Lima, Renata Marques David, Ercy Mara Cipulo Ramos

Universidade Estadual Paulista - FCT/UNESP. Departamento de Fisioterapia, Presidente Prudente - SP. E-mail: camila aguiar2003@yahoo.com.br - Órgão de fomento: Fundação de Amparo à Pesquisa do Estado de São Paulo (FAPESP).

\section{RESUMO}

Indivíduos tabagistas possuem importantes mudanças sistêmicas, sendo elas influenciadas principalmente pela nicotina presente no cigarro que induz a produção de catecolaminas ao nódulo sino-atrial gerando um aumento da frequência da cardíaca $(\mathrm{FC})$ e pressão arterial sistêmica (PA). O monóxido de carbono (CO) prejudica a oxigenação dos tecidos e pouco se sabe sobre esses efeitos à longo prazo. Desta forma, o objetivo deste estudo foi correlacionar o tempo de tabagismo com o comportamento dos sinais vitais: $\mathrm{FC}$, saturação periférica de oxigênio $\left(\mathrm{SpO}_{2}\right), \mathrm{PA}$, percepção subjetiva de dispneia (Borg $D$ ) e percepção subjetiva de fadiga em membros inferiores (Borg F). Participaram 40 indivíduos tabagistas $(44,55 \pm 13,70$ anos) aparentemente saudáveis. Não houve correlação entre FC, PAS, PAD e Borg com o tempo de tabagismo, porém a $\mathrm{SpO}_{2}$ apresentou uma correlação negativa com o tempo, indicando um maior prejuízo na oxigenação periférica de indivíduos com um maior tempo de tabagismo.

Palavras - chave: tabagismo, frequência cardíaca, pressão arterial, saturação de oxigênio, sinais vitais.

\section{INFLUENCE OF DURATION OF SMOKING IN VITAL SIGNS}

\begin{abstract}
Individuals smokers have important systemic changes, found in cigarettes nicotine induces the production of catecholamines sino-atrial node that generates an increased frequency of heart (HR) and blood pressure (BP). Therefore, the objective of this study was to correlate the duration of smoking with the behavior of vital signs: HR, peripheral oxygen saturation (SpO2), PA, subjective perception of dyspnea (Borg $D$ ) and subjective perception of fatigue in the lower limbs (F Borg). Forty apparently healthy smokers $(44,55 \pm 13,70$ years). There was no correlation between HR, SBP, $\mathrm{DBP}$ and Borg with the duration of smoking, but the $\mathrm{SpO} 2$ showed a negative correlation with time, indicating a greater loss in peripheral oxygenation of patients with longer duration of smoking.
\end{abstract}

Keywords: smoking, heart rate, blood pressure, oxygen saturation, vital signs. 


\section{INTRODUÇÃO}

O tabagismo é um dos maiores problemas de saúde pública, sendo considerada pela Organização Mundial da Saúde (OMS) a principal causa de morte evitável no mundo. Apesar da crescente implementação de programas de cessação tabagística, o número de dependentes do tabaco ainda é alto, visto que um terço da população mundial adulta é fumante ${ }^{1-3}$.

A fumaça do cigarro contém mais de 4.700 substâncias tóxicas, incluindo o alcatrão, arsênico, amônia, corantes, agrotóxicos em altas concentrações, além de monóxido de carbono (CO) e nicotina, sendo esses dois últimos os principais compostos tóxicos. Todas essas substâncias são inaladas e favorecem o desenvolvimento de doenças tabaco relacionadas, como cânceres, diabetes, doenças cardiovasculares e respiratórias crônicas ${ }^{2-4}$.

Os principais efeitos maléficos causados no organismo têm como responsável o CO. Devido à alta afinidade do sangue com o $\mathrm{CO}$, a oxigenação dos tecidos é prejudicada. Ainda, já se sabe que o hábito de fumar é capaz de reduzir em até $12 \%$ a capacidade aeróbia do indivíduo ${ }^{5-6}$. Além disso, o consumo de um cigarro causa um aumento efetivo de $14 \%$ na frequência cardíaca $(\mathrm{FC})$ e de $6 \%$ na pressão arterial $(P A)^{7}$. Essa reação se deve, provavelmente, ao aumento das concentrações de adrenalina e noradrenalina plasmáticas durante $\mathrm{o}$ período em que se fuma ${ }^{8}$. Os níveis de norepinefrina aumentam em 12,5 minutos, com um pico em 15 minutos, retornando aos níveis iniciais após 30 minutos. Essa alteração ocasiona o aumento máximo da PA e, passados 30 minutos, ocorre queda desses valores, permanecendo, no entanto, mais elevados que os valores registrados antes do ato de fumar ${ }^{8}$.

Além do $\mathrm{CO}$, a nicotina também tem papel central nos efeitos nocivos causados pelo uso do cigarro. De acordo com Rebelo et. al. ${ }^{9}$ após a inalação da fumaça do cigarro, a nicotina chega ao cérebro de 10 a 16 segundos e atua durante cerca de duas horas. A ação da nicotina se faz fundamentalmente através do sistema nervoso autônomo ${ }^{10}$, aumentando a FC, devido ao estímulo das catecolaminas ao nódulo sino-atrial, e também conduz ao aumento no débito cardíaco e volume de ejeção, assim como vasoconstrição, elevando a PA. Tais efeitos nocivos causados em decorrência do uso do tabaco/cigarro podem se agravar com o tempo.

Erdem et al $^{11}$ sugerem que 0 tabagismo contribui para o comprometimento do sistema autonômico cardiovascular em adultos fumantes de longo prazo e apontam efeitos no coração diante da exposição crônica ao cigarro. 
Contudo, percebe-se uma carência de estudos com a análise da correlação existente entre o tempo de tabagismo e os prejuízos sistêmicos. Sendo assim, o objetivo do presente estudo foi correlacionar os anos de tabagismo com os sinais vitais: FC, PA, saturação parcial de oxigênio $\left(\mathrm{SpO}_{2}\right)$ e percepção subjetiva de esforço em repouso de indivíduos tabagistas.

\section{MÉTODOS}

O presente estudo é de natureza transversal, no qual foram avaliados indivíduos tabagistas ativos, que faziam uso de cigarro comum, de ambos os gêneros e com idade superior a 18 anos.

Todos os participantes foram instruídos a respeito dos métodos de avaliação e assinaram o termo de consentimento livre-esclarecido. O estudo foi aprovado pelo comitê de ética em pesquisa da FCT-Unesp, campus de Presidente Prudente. Processo no 18/2011.

Inicialmente, todos os voluntários realizaram uma avaliação inicial onde responderam a uma entrevista fornecendo informações sobre diversos aspectos: dados pessoais, história do tabagismo (anos de tabagismo, tipo de cigarro) e história clínica (foram questionados se apresentavam alguma doença diagnosticada por médico).

Os indivíduos aparentemente saudáveis, que não apresentavam doenças, foram agendados para o segundo dia de avaliação, e para esta foram orientados a não ingerirem substâncias à base de cafeína por pelo menos 4 horas antes da avaliação e estarem em abstinência de 12 horas entre o último cigarro e o início da avaliação. Neste segundo momento, os indivíduos permaneceram em repouso durante 20 minutos para que os parâmetros cardiovasculares se estabilizassem, e então foram avaliados quanto a: FC que foi determinada pelo cardiofrequencímetro Polar S810i; $\mathrm{A} \mathrm{SpO}_{2}$ foi verificada por meio de oxímetro da marca $\mathrm{BCl}$ 3303; PA analisada pelo método auscultatório no membro superior esquerdo, utilizando esfigmomanômetro aneróide e estetoscópio da marca BIC; por fim foram questionados sobre a percepção subjetiva de dispneia (Borg D) e percepção subjetiva de fadiga em membros inferiores (Borg F).

Os dados foram analisados pelo programa estatístico GraphPadPrism 5. Para avaliar a normalidade dos dados foi realizado o teste de Shapiro-Wilk. Para a correlação foi utilizado o teste de Pearson para dados normais e o teste de Spearman para dados não normais. O nível de significância utilizado foi de $p<0,05$

\section{RESULTADOS}

A amostra foi composta por 40 indivíduos. As características 
clínicas e as variáveis que

estão apresentadas na Tabela 1.

demonstram a carga tabagística

Tabela 1. Caracterização da amostra.

\begin{tabular}{lc}
\hline & Participantes $(\mathbf{n}=\mathbf{4 0})$ \\
\hline Sexo $(\mathrm{M} / \mathrm{F})$ & $16 / 24$ \\
Idade $(\mathrm{anos})$ & $44,55 \pm 13,70$ \\
IMC $\left(\mathrm{kg} / \mathrm{m}^{2}\right)$ & $24,95 \pm 4,152$ \\
Anos que fuma & $25,90 \pm 12,69$ \\
\hline
\end{tabular}

Legenda: IMC: Índice de massa corporal.

Na tabela 2 encontra-se os valores, sinais vitais ( $F C, P A S, P A D, \mathrm{SpO}_{2}$ ) e percepção expressos em média e desvio padrão, dos subjetiva de esforço (Borg).

Tabela 2. Valores de sinais vitais em média e desvio padrão.

\begin{tabular}{llc}
\hline Variáveis & Média e desvio padrão & Valor de $\mathbf{p}$ \\
\hline FC & $72,98 \pm 8,83$ & 0,36 \\
$\mathrm{PAS}$ & $119,3 \pm 14,03$ & 0,06 \\
$\mathrm{PAD}$ & $83,50 \pm 19,42$ & 0,38 \\
$\mathrm{SpO}_{2}$ & $97,08 \pm 1,66$ & $0,015^{*}$ \\
Borg D & $0,46 \pm 0,87$ & 0,96 \\
Borg F & $7,0 \pm 2,09$ & 0,67
\end{tabular}

Legenda: FC: frequência cardíaca; PAS: pressão arterial sistólica; PAD: pressão arterial diastólica, $\mathrm{SpO}_{2}$ : saturação periférica de oxigênio, Borg D: percepção subjetiva de dispneia, Borg F: percepção subjetiva de fadiga em membros inferiores.

Abaixo, na figura 1, encontra-se as correlações entre anos que fuma e FC, PAS, PAD, Borg D e Borg $F$, no qual se observa uma correlação negativa entre $\mathrm{SpO}_{2}$ e anos que fuma ( $p=0,015$ e $r=-0,37)$, mostrando que quanto maior o tempo que o indivíduo fuma, mais prejudicada se encontra a $\mathrm{SpO}_{2}$ indicando assim uma possível oxigenação prejudicada.

Analisando as outras variáveis, não foi encontrada diferença estatística significante. 

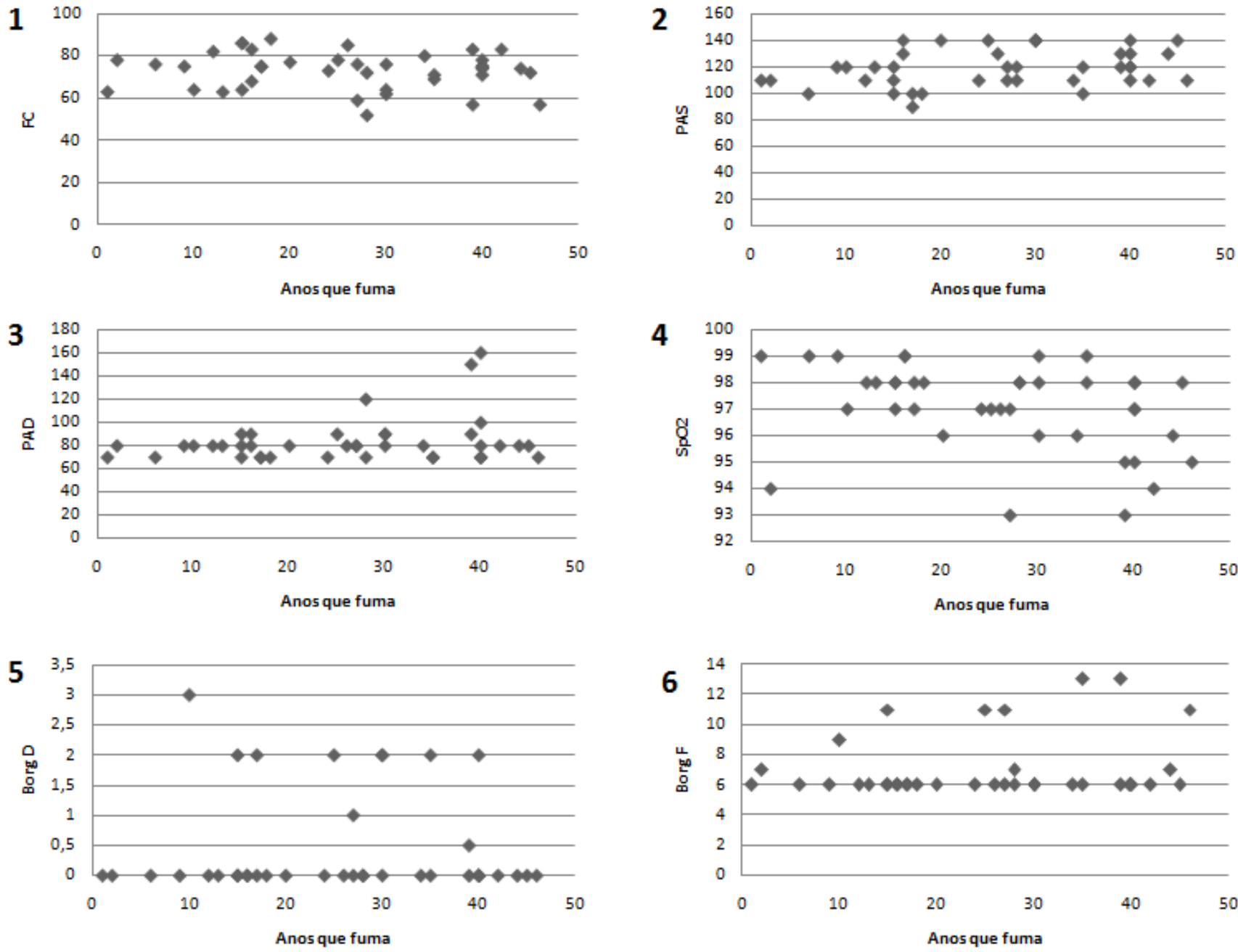

Figura 1. Gráficos de dispersão apresentando correlações entre anos que fuma e as seguintes variáveis: frequência cardíaca (gráfico 1), pressão arterial sistólica (gráfico 2), pressão arterial diastólica (gráfico 3), saturação periférica de oxigênio (gráfico 4), Borg de dispneia (gráfico 5) e Borg de fadiga em membros inferiores (gráfico 6).

\section{DISCUSSÃO}

De acordo com os resultados, a amostra apresentou diferença estatística apenas na $\mathrm{SpO}_{2}$, mostrando uma correlação negativa entre anos que fuma, isto é, - quanto maior o tempo de tabagismo, maior o prejuízo da oxigenação periférica dos tecidos. Evidências ${ }^{12}$ mostram que fumar compromete a oxigenação dos tecidos devido aos níveis

aumentados

de carboxihemoglobina, em resultado da exposição ao monóxido de carbono existente no fumo. Uma eritrocitose compensatória pode ser encontrada em alguns fumantes, em particular quando o consumo do tabaco é elevado. Em resultado de todos esses mecanismos, os fumantes apresentam uma menor capacidade de transporte de 
oxigênio e um comprometimento da circulação periférica, causado pelo aumento da viscosidade sanguínea e da leucocitose.

Entretanto, a FC, PAS e PAD não apresentaram diferença estatística significante quando correlacionados com o tempo de tabagismo. Esses dados se mostram contraditórios quando comparado a outros estudos $^{7,10}$. Este achado pode se dar ao fato do tempo de abstinência de 12 horas que os indivíduos tabagistas se encontravam no momento da avaliação.

Estudos afirmam a influência do tempo de abstinência do cigarro no comportamento da FC. Myrstenet et al. ${ }^{13}$ detectaram uma redução na $\mathrm{FC}$ em um curto período de abstinência (5 minutos), em indivíduos de ambos os sexos. Resultados semelhantes são encontrados em estudos com maiores períodos de abstinência.

Goldbarg et al. ${ }^{14}$ detectaram uma diminuição na $F C$ em um período de abstinência de 30 minutos. O efeito da diminuição da FC se dá, provavelmente, pela diminuição da nicotina circulante causada pela redução das catecolaminas ao nódulo sino-atrial, o que também afeta, segundo o estudo de Rebelo et al. ${ }^{9}$, a PA.

Ainda, Minamiet et al. ${ }^{15}$ mostraram que as percepções subjetivas de esforço se reduzem após um período de 24 horas de abstinência do cigarro, o que também pode justificar a ausência de correlação no presente estudo.

Dessa, forma o presente mostrou que o tempo de abstinência do último cigarro até o dia da avaliação influenciou nos resultados encontrados, indicando a possibilidade de diferentes abordagens para futuros estudos relacionadas ao tema.

\section{CONCLUSÃO}

Assim, conclui-se que houve uma correlação negativa entre o tempo de tabagismo e a $\mathrm{SpO}_{2}$ e que a mesma não foi vista quando realizada com outras variáveis (FC, PAD, PAS, Borg D e Borg F).

\section{REFERÊNCIAS}

1. BRASIL. MINISTÉRIO DA SAÚDE. INSTITUTO NACIONAL DE CÂNCER - INCA. Abordagem e Tratamento do Fumante Consenso 2001. Rio de Janeiro: INCA, 2001. $38 p$.

2. Who Report On The Global Tobacco Epidemic, Warning about the dangers of tobacco. 2011.

3. Rodrigues ESR, Cheik NC, Mayer AF. Nível de atividade física e tabagismo em universitários. Rev Saúde Pública. 2008;42(4):672-8.

http://dx.doi.org/10.1590/S0034$\underline{89102008000400013}$

4. Niedermaier ON, Smith ML, Beightol LA, Zukowska-Grojec Z, Goldstein DS, Eckberg DL. Influence of cigarette smoking on human autonomic function. Circulation. 
1993;88(2):562-571.

http://dx.doi.org/10.1161/01.CIR.88.2.562

5. Garcia ES, Teixeira MM. Efeitos do cigarro, da nicotina e do monóxido de carbono sobre alguns parâmetros fisiológicos: uma revisão da literatura. Rev Bras Ciênc Mov. 1989;3(2):32-9.

6. Costa $A A$, Elabras Filho J, Araújo ML, Ferreira JES, Meirelles LR, Magalhães CK. Programa multiprofissional de controle do tabagismo: aspectos relacionados à abstinência de longo prazo. Revista da SOCERJ. 2006;19(5):397-403.

7. Kool MJ, Heks AP, StruigkerBudier HA. Short and long-term effects ofsmoking on arterial wall properties in habitual smokers. J Am Coll Cardiol1993; 22: 1881-86. http://dx.doi.org/10.1016/07351097(93)90773-T

8. Cryer PE, Haymond MW, Santiago JV, Shah SD. Norepinephrine andepinephrine release and adrenergic mediation of smoking-associatedhomodynamic and metabolic events. N Engl J Med 1976; 295: 573-77.

http://dx.doi.org/10.1056/NEJM1976090929 $\underline{51101}$

9. Rebelo L. Tabaco, cérebro e dependência: cérebro toxicodependente. Rev Port Clín Geral.2006;22(1):197-9.

10. Franken RA, Nitrini $G$, Franken $M$, Fonseca AG, Leite JTC. Nicotina. ações e interações. Arq Bras Cardiol. 1996;66(1):3713.

11. Erdem A, Ayhan SS, Ozturk S, Ozlu MF, Alcelik A, SahinS,Tosun T, Erdem FH, Gumustekin K, YaziciM.Cardiac autonomic function in healthy young smokers. Toxicology and Industrial Health. ToxicollndSaúde. 2012; DOI:10.1177 / 0748233712468024.
12. U.S. Department of Health and Human Services. The health consequences of smoking:

a report of the Surgeon General.Atlanta: U.S.Department of Health and Human Services, Centers for Disease Control and Prevention, National Center for Chronic Disease Preventionand Health Promotion, Office on Smoking and Health; 2004

13. Myrsten AL. Elgerot A, Edgren B. Effects of abstinence from tobacco smoking on physiological and psychological arousal levels in habitual smokers. Psychosom Med.1977;39(1)25-38.

http://dx.doi.org/10.1097/00006842-

197701000-00004

14. Goldbarg A, Krone R, Resnekov L. Effects of cigarette smoking on hemodynamics at rest and during exercise in normal subjects. Chest. 1971;60(6):531-6. http://dx.doi.org/10.1378/chest.60.6.531

15. Minami J, Ishimitsu $T$, Matsuoka $H$. Effect of smoking cessation on Blood Pressure and heart rate variability in habitual smokers. Hypertension. 1999;33(1 Pt 2):58690.

http://dx.doi.org/10.1161/01.HYP.33.1.586

Recebido para publicação em 12/08/2014

Revisado em 28/08/2014

Aceito em 01/09/2014 